\title{
Variaciones espaciales y ontogenéticas en la dieta de un plecóptero de amplia distribución Claudioperla tigrina (Plecoptera: Gripopterygidae)
}

\author{
María Celina Reynaga, Natalia Dávalos \& Carlos Molineri \\ Instituto de Biodiversidad Neotropical, CONICET (Consejo Argentino de Investigaciones Científicas y Técnicas), \\ Facultad de Ciencias Naturales e IML, Universidad Nacional de Tucumán, Miguel Lillo 205, CP 4000, San Miguel \\ de Tucumán, Argentina; celina_reynaga@yahoo.com, natidavalos83@hotmail.com, carlosmolineri@gmail.com
}

Recibido 18-IV-2016. Corregido 24-II-2017. Aceptado 23-III-2017.

\begin{abstract}
Spatial and ontogenetic variations in the diet of a widely distributed stonefly Claudioperla tigrina (Plecoptera: Gripopterygidae). Dietary information gives insight into several ecological processes acting in lotic ecosystems. This work aimed: 1) to identify the dietary habits of Claudioperla tigrina immature stages along a wide altitudinal as well as latitudinal gradient in North Argentina; 2) to define the functional feeding group (FFG) of C. tigrina; 3 ) to evaluate differences in diet in the studied sites. Studied nymphs were collected from localities widely scattered in Northwestern Argentina and they fell into different developmental stages (four size classes). The ingested material was extracted from the foregut and midgut by using thorax ventral dissection. Dietary profiles were analyzed through the estimation of parameters associated with a Dirichletmultinomial distribution. ANOVA's were performed for each food item using sites as factor. Multidimensional Scaling was used to identify sites with similar dietary profiles. An analysis of food-niche breadth was also performed to evaluate the degree of dietary diversification for the resources consumed in each site. Mouthparts are similar across the different size classes, excepting the increasing sclerotization recorded with age. Mouthparts retained most of the typical chewing groundplan, showing relatively short labial and maxillar palps, and strong, sclerotized and denticulated mandibles and maxillae. Our results pointed out that the nymphs of C. tigrina always ingest two or more food items (CPOM, FPOM, invertebrates and algae), suggesting thus a flexible diet. The diet changed in relation to body size, while finer particles were consumed in the early stages, larger particles were ingested in final stages. Coarse particulate organic matter was the dominant food item, with signals of shredding during ingestion. Differences between sites for FPOM, invertebrates, algae and sediment were detected, but not for MOPG. Correlations were obtained for the first two axis of the MDS analysis. Sites AP, LT, LI, C and M (Yungas Rainforest and Humid Grassland) were negatively correlated with the axis 1 which was associated with increased consumption of FPOM. On the positive side of the axis the site P (High Andes) is associated with a greater proportion of invertebrates and sediment. The sites IN (Humid Grassland) and LR (Argentine Northwest Monte and Thistle of the Prepuna) were located at the positive domain of axis 2 which is in turn associated with a greater count of algae in the dietary contents. We found significant differences in the quantity of secondary items, and this is likely related with the resources environmental availability. The FFG of Claudioperla tigrina is primarily shredder/collector-gatherer in Yungas Rainforest and Humid Grassland shredder/predator in High Andes. FFG classification of C. tigrina and the definition of their role for organic matter processing is an important step for future studies based on functional groups such as analysis of food webs. Rev. Biol. Trop. 65 (3): 1174-1184. Epub 2017 September 01.
\end{abstract}

Key words: functional feeding groups, gut content analysis, Yungas Rainforest, Humid Grasslands, High Andes, Argentine Northwest Monte and Thistle of the Prepuna.

Los insectos acuáticos ejercen numerosos roles relacionados con la actividad biológica de las aguas corrientes como puede ser la descomposición de la materia orgánica y su transformación en heces, afectando a las propiedades físicas y químicas de los sistemas lóticos (Bello \& Cabrera, 2001). El conocimiento de los hábitos alimentarios constituye una pieza clave para comprender tanto la estructura de la comunidad de invertebrados como el flujo de energía 
del sistema que la contiene (Cummins, 1973; Albariño \& Díaz Villanueva, 2006; Reynaga \& Rueda Martín, 2014).

El enfoque de grupo funcional trófico (GFT) está basado en la asociación entre los mecanismos morfológicos, comportamiento de adquisición del alimento y el tipo de alimento ingerido. Siguiendo este enfoque, los insectos acuáticos se clasifican en las categorías de colectores (recolectores y filtradores), trituradores, raspadores y depredadores (Merrit \& Cummins, 1996). Esta clasificación funcional fue desarrollada para el hemisferio norte, y se encuentra en proceso de ajuste para insectos acuáticos de ríos argentinos de bajo orden (Wais, 1990; Albariño \& Valverde, 1998; Díaz Villanueva \& Albariño, 1999; Albariño, 2001; Albariño, Díaz Villanueva, \& Canhoto, 2008; Reynaga \& Rueda, 2008; Reynaga, 2009; Reynaga \& Rueda Martín, 2014).

El orden Plecoptera presenta cerca de 3 500 especies descritas, cuyos estados inmaduros se encuentran asociados a cursos de aguas corrientes bien oxigenados (Fochetti \& De Figueroa, 2008). Sus ninfas acuáticas cumplen un importante rol en las cadenas tróficas, encontrándose en diversos sistemas hídricos continentales (Castillo, Zúñiga, \& Bacca, 2013). En general, las ninfas consumen una amplia gama de alimentos donde el tipo de alimento ingerido depende de la especie, estado del desarrollo, hora del día y disponibilidad de recursos (Tamarís-Turizo, TurizoCorrea, \& Zúñiga, 2007; Stark, Froehlich, C., \& Zúñiga 2009).

Para el Noroeste de Argentina (NOA) se han citado hasta el presente tres géneros de plecópteros: Anacroneuria Klapálek 1909 (Perlidae), Kempnyia Klapálek 1914 (Perdidae) y Claudioperla Klapálek 1904 (Gripopterygidae). El GFT de Anacroneuria fue determinado por Tomanova, Goitia y Helesic (2006) y Reynaga y Dos Santos (2012) como depredador; Kempnyia también fue definido como depredador por Righi-Cavallaro, Froehlich y Cavallaro (2014), mientras que para Claudioperla no fue asignado un GFT particular.
Claudioperla posee tres especies descritas en Bolivia (Gibon \& Molina, 2013), pero solo C. tigrina se encuentra también en Argentina, desde el noroeste hasta la Patagonia (Pessacq, 2009). En el presente trabajo se pretende: 1) aportar al conocimiento de los hábitos alimentarios de las ninfas de Claudioperla tigrina a partir de muestras recolectadas en un amplio gradiente altitudinal y latitudinal en Argentina; 2) asignar a la especie un grupo funcional trófico basándose principalmente en el análisis de contenido del tracto digestivo y morfología de su aparato bucal; 3) evaluar diferencias en la alimentación en los sitios estudiados.

\section{MATERIALES Y MÉTODOS}

El estudio se realizó con material recolectado en ocho arroyos andinos de bajo orden, de tres provincias del NOA (Fig. 1): Salta, Tucumán y La Rioja, cuyas altitudes varían entre 1 645 y 4467 msnm (Cuadro 1), pertenecientes a cuatro ecorregiones según la clasificación de Olson et al. (2001). Estos arroyos poseen aguas limpias, claras, oligotróficas, sin contaminación aparente. Los mayores caudales ocurren en primavera-verano (noviembre a marzo) y los menores en otoño-invierno (abril a octubre). Las muestras pertenecientes a los sitios AP, M y LR fueron tomadas en período de aguas bajas, el resto corresponde a épocas de aguas altas.

En cada sitio y ocasión de muestreo (Cuadro 1), se tomaron tres muestras cualitativas en las que se colectaron especímenes de ninfas de C. tigrina con redes entomológicas (Surber, de pateo, y de mano) de variada abertura de malla (0.3-1.0 mm). Los mismos fueron separados, determinados, medidos y diseccionados bajo lupa estereoscópica Olympus SZ 61 de 40X. Las ninfas fueron identificadas como C. tigrina mediante su cría hasta el estado adulto. Los imagos machos fueron determinados siguiendo las descripciones e ilustraciones disponibles para la especie (Klapálek, 1904; Illies, 1963; Stark, Kondratireff, \& Gill, 2012; Gibon \& Molina, 2013). Se establecieron cuatro clases de tamaño para las ninfas según la longitud 


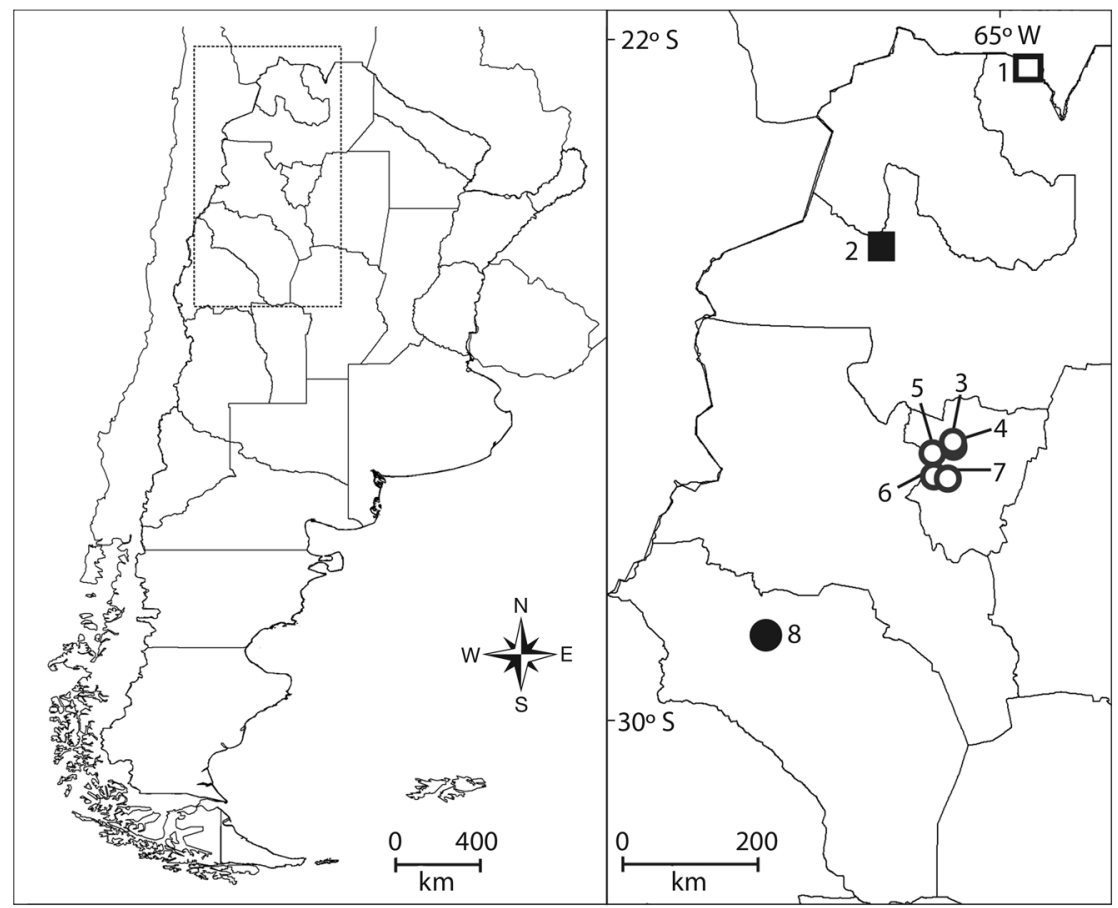

Fig. 1. Área de estudio indicando los puntos de muestreo en las ecorregiones consideradas: Altos Andes (घ), Pastizales de Neblina (०), Selva de Yungas ( $\square$ ) y Monte de Sierras y Bolsones $(\bullet)$.

Fig. 1. Study area with sampling points projected in ecoregions: High Andes ( $\bullet$ ), Humid Grasslands (०),Yungas Rainforest $(\square)$ and Argentine Northwest Monte and Thistle of the Prepuna $(\bullet)$.

total del cuerpo (no incluye apéndices): 1) < $3.9 \mathrm{~mm}$, 2) 4 a $5.9 \mathrm{~mm}, 3) 6$ a $7.9 \mathrm{~mm}, 4)$ $>$ de $8 \mathrm{~mm}$. Las clases de tamaño no se corresponden con estadios ninfales, ya que éstos son de número variable e indeterminado para la especie en estudio. Se realizaron disecciones de las piezas bucales para cada clase de tamaño con el objeto de detectar posibles diferencias en el aparato bucal. Todo el material se encuentra depositado en la colección entomológica del Instituto de Biodiversidad Neotropical (Tucumán, Argentina) y conservado en alcohol $96^{\circ}$.

Análisis de dieta: El análisis de contenido alimentario se efectuó siguiendo la metodología propuesta por Reynaga y Rueda Martín (2008). Se realizaron disecciones ventrales de ejemplares de todas las clases de tamaño para cada localidad en estudio. Se extrajo la parte anterior del tracto digestivo correspondiente a los tres segmentos torácicos. El contenido estomacal de cada ejemplar fue montado por separado en glicerina sobre una superficie de vidrio con una dimensión de $1 \times 1 \mathrm{~cm}$ para facilitar su observación y recuento bajo microscopio óptico Olympus BX 51 con un aumento de 100X. Los ítems alimentarios se clasificaron en seis categorías: 1) materia orgánica particulada fina (MOPF) $<1 \mathrm{~mm}$; 2) materia orgánica particulada gruesa (MOPG) $>1 \mathrm{~mm} ; 3$ ) algas (A); 4) invertebrados (I); 5) sedimentos (S); y 6) no identificado (NI). Por cada ejemplar, se analizaron 10 transectas del campo óptico elegidas al azar, y cada una de ellas incluyó diez puntos de observación, con un intervalo constante entre ellas, correspondientes a la escala micrométrica del microscopio, y contando un único ítem alimentario por punto.

Se analizaron los perfiles dietarios mediante la estimación de parámetros de distribución 


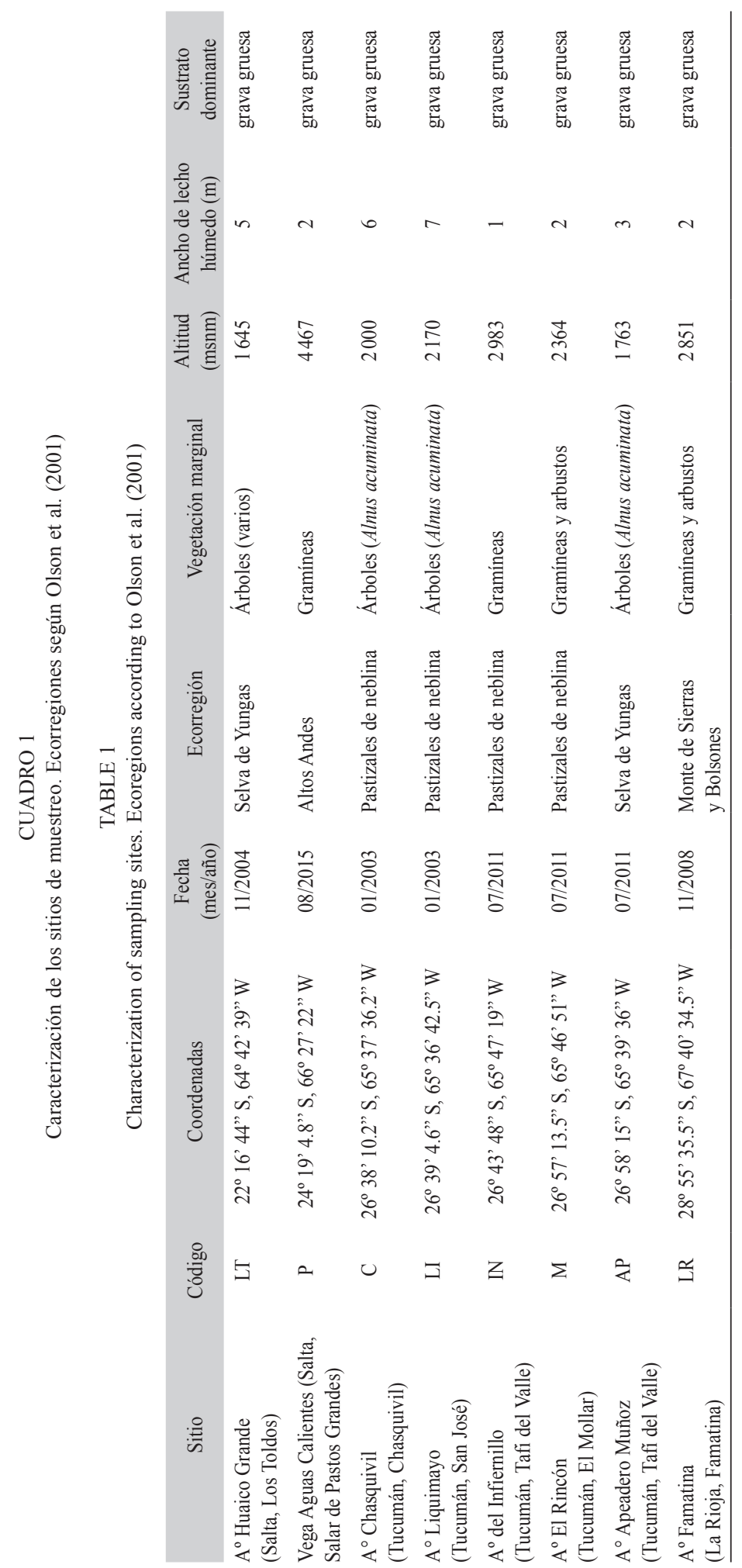


Dirichlet-multinomial, utilizando el paquete dirmult: Estimation in Dirichlet-Multinomial distribution ( $\mathrm{R}$ program, Tvedebrink, 2015). Se efectuaron ANOVA's para cada rubro alimentario en función de los sitios y las comparaciones de pares se hicieron mediante el procedimiento de Tukey. En este estudio, se utilizó la técnica Multidimensional Scaling (MDS) para identificar los sitios con perfiles alimentarios promedios similares. Se empleó la distancia de Aitchison (Aitchison \& Greenacre, 2002), siendo la más apropiada para datos composicionales, como efectivamente lo son los datos de dieta.

Se determinó la amplitud de nicho trófico (diversificación del recurso consumido) en cada sitio según lo propuesto por De Cáceres, Sol, Lapiedra y Legendre (2011):

$$
B_{\text {pop }}=(1 / 2) \Sigma_{\mathrm{j}=1}^{\mathrm{r}} \Sigma_{\mathrm{k}=1}^{\mathrm{r}} f_{j} f_{k} d_{j k}
$$

Realizamos nuestros cálculos sin tener en cuenta la similitud entre los recursos (es decir que $d_{j k}=1$ en todos los casos). Para determinar el intervalo de confianza $95 \%$, se empleó la técnica estadística de bootstrap, aplicando 1000 repeticiones de muestras extraídas a partir de una distribución multinomial con el vector de probabilidades correspondiente al perfil dietario representativo de cada sitio.

\section{RESULTADOS}

Se analizaron en total 115 individuos entre las cuatro clases de tamaño consideradas. Las ninfas de la clase de tamaño 1 presentan mandíbulas con tres incisivos bien diferenciados y palpos labiales que carecen de una clara segmentación. Las ninfas de la clase de tamaño 2, 3 y 4 presentaron mandíbulas bien desarrolladas con cuatro incisivos agudos, fuertes, bien delimitados y molar portador de numerosos dentículos. Las maxilas cuentan con lacinia y gálea de tamaño semejantes y palpos tetrasegmentados y pilosos. La lacinia se encuentra terminada en dos puntas esclerosadas y con numerosos dientes alargados en el margen interno distribuidos en una hilera de aproximadamente 10 dientes; la gálea está poco esclerosada con escasos y diminutos dentículos apicales. El labio posee un prementón definido, glosa y paraglosa de igual longitud, los palpos labiales tienen tres segmentos bien delimitados, cortos y robustos, cubiertos de setas. El labro presenta setas largas en su margen anterior. En general, la esclerotización y el número de setas aumentan con el tamaño de las ninfas.

El contenido del tracto digestivo consistió de una variada gama de alimentos (Cuadro 2). Los ítems alimentarios dominantes fueron en todos los casos MOPG y MOPF. Como MOPG

CUADRO 2

Composición dietaria y medidas morfológicas para las cuatro clases de tamaño consideradas de C. tigrina

TABLE 2

Dietary composition and morphological measurements for the classes of $C$. tigrina considered

\begin{tabular}{cccccccccccc}
$\begin{array}{c}\text { Clases de } \\
\text { tamaño }\end{array}$ & $\begin{array}{c}\mathrm{N}^{0} \text { de } \\
\text { Individuos }\end{array}$ & \multicolumn{2}{c}{$\begin{array}{c}\text { Longitud del } \\
\text { cuerpo }(\mathrm{mm})\end{array}$} & \multicolumn{2}{c}{$\begin{array}{c}\text { Ancho de } \\
\text { cabeza }(\mathrm{mm})\end{array}$} & MOPF & MOPG & I & A & S & NI \\
1 & 15 & 3.25 & $(0.15)$ & 0.66 & $(0.04)$ & $5.6(0.98)$ & $4.2(0.51)$ & $0.6(0.27)$ & $1.2(0.58)$ & $0.4(0.16)$ & $0(0)$ \\
2 & 34 & 4.98 & $(0.1)$ & 0.98 & $(0.03)$ & $11.38(1.3)$ & $12.15(1.81)$ & $3.09(0.84)$ & $2.26(0.54)$ & $0.47(0.18)$ & $0.41(0.25)$ \\
3 & 40 & 6.78 & $(0.09)$ & 1.11 & $(0.02)$ & $15.83(1.64)$ & $16(1.52)$ & $5.32(1.18)$ & $2.54(0.55)$ & $0.49(0.22)$ & $0.27(0.1)$ \\
4 & 25 & 8.43 & $(0.06)$ & 1.26 & $(0.03)$ & $20.21(2.89)$ & $20.67(2.13)$ & $2.96(1.16)$ & $2.71(0.76)$ & $0.13(0.09)$ & $0.71(0.19)$ \\
\hline
\end{tabular}

Se informa el valor promedio con su respectivo error estándar entre paréntesis. La frecuencia de los items alimenticios está expresada como promedio de los eventos de conteo realizados durante el muestreo puntual del contenido estomacal. MOPF: materia orgánica particulada fina, MOPG: materia orgánica particulada gruesa, I: invertebrado, A: algas, S: sedimento, NI: no identificado.

The average value with its respective standard error is reported in brackets. The frequency of food items is expressed as average counts events performed during the point sampling of stomach contents. FPOM: fine particulate organic matter, MOPG: coarse particulate organic matter, I: invertebrate, A: algae, S: sediment, NI: unidentified. 
se identificaron hojas, pólen, tricomas y raíces. Entre los fragmentos de invertebrados se encontraron escleritos indeterminados y pocas estructuras reconocibles pertenecientes a larvas de Chironomidae (Diptera), mandíbulas y maxilas de Leptophlebiidae (Ephemeroptera), y uñas y antenas de $C$. tigrina. Se observó la presencia de fragmentos de alga Spirogyra (Zygnemataceae) entre otras algas no determinadas. Las cantidades de sedimento y material no identificado fueron muy bajas y poco relevantes.

Se graficó mediante un diagrama de barras el perfil dietario para cada sitio estudiado (Fig. 2). Se efectuaron los ANOVA's para cada rubro alimentario (Cuadro 3). Las comparaciones por pares se hicieron mediante el procedimiento de Tukey y se usó la denotación sintética por letras (diferentes letras $=$ diferencias significativas) sobre diagramas de caja realizados para cada rubro (Fig. 3). Se encontraron diferencias significativas entre sitios $(\mathrm{P}<0.001)$ para los ítems MOPF, invertebrados, algas y sedimento, pero no para MOPG. En los sitios AP, C, M, Li y LT se registra de forma significativa el ítem MOPF, en el sitio P y LR los restos de invertebrados y sedimento, y en el sitio IN la presencia de algas.

El análisis MDS dio un resultado bidimensional sobre la base de los perfiles promedios (Fig. 4). Se realizaron correlaciones para cada eje $(\mathrm{P}<0.05)$. Para el eje 1 se agruparon en el lado negativo los sitios AP, LT, LI, C y M (Selva de Yungas y Pastizal de neblina) que se correspondieron con un mayor consumo de

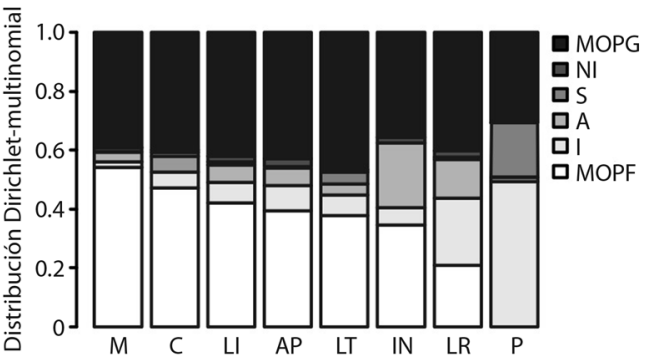

Fig. 2. Perfil representativo de la dieta de C. tigrina por sitio de estudio según distribución multinomialDirichlet. Sitios: $A^{\circ}$ El Rincón (M), $A^{\circ}$ Chasquivil (C), $\mathrm{A}^{\mathrm{o}}$ Liquimayo (LI), $\mathrm{A}^{\mathrm{o}}$ Apeadero Muñoz (AP), $\mathrm{A}^{\mathrm{o}}$ Huaico Grande (LT), $\mathrm{A}^{\mathrm{o}}$ del Infiernillo (IN), $\mathrm{A}^{\mathrm{o}}$ Famatina (LR), Vega Aguas Calientes (P). Ítems alimentarios: Materia orgánica particulada gruesa (MOPG), no identificado (NI), sedimento (S), algas (A), invertebrados (I), materia orgánica particulada fina (MOPF).

Fig. 2. Representative profile of the diet of C. tigrina by study site according to multinomial-Dirichlet distribution. Sites: $A^{\circ}$ El Rincón (M), $A^{\circ}$ Chasquivil (C), $A^{\circ}$ Liquimayo (LI), $A^{\circ}$ Apeadero Muñoz (AP), $A^{\circ}$ Huaico Grande (LT), $\mathrm{A}^{\mathrm{o}}$ del Infiernillo (IN), $\mathrm{A}^{\mathrm{o}}$ Famatina (LR), Vega Aguas Calientes (P). Food items: coarse particulate organic matter (MOPG), unidentified (NI), sediment (S), algae (A), invertebrate (I), fine particulate organic matter (MOPF).

MOPF $(R=-0.94, t=-6.74)$, en el lado positivo del eje se encontró el sitio P (Altos Andes) asociado a los ítems invertebrados $(\mathrm{R}=0.98, \mathrm{t}$ $=11.59)$ y sedimento $(\mathrm{R}=0.96, \mathrm{t}=8.54)$. Para el eje 2 se estableció una asociación positiva de los sitios IN (Pastizal neblina) y LR (Monte de Sierras y Bolsones) con la presencia de algas $(\mathrm{R}=0.89, \mathrm{t}=4.83)$. El ítem dominante en todos los casos corresponde a la MOPG,

CUADRO 3

Resumen de los ANOVA's efectuados para cada rubro alimentario en función de los sitios estudiados

TABLE 3

Summary of ANOVA's carried out for each food item according to the sites studied

\begin{tabular}{lcccc}
\multicolumn{1}{c}{ Fuente de variación } & Suma de cuadrados & Cuadrados medios & $F(7,106)$ & $\operatorname{Pr}(>F)$ \\
MOPF & 1.971 & 0.2816 & 13.6 & $>0.001$ \\
MOPG & 0.201 & 0.0287 & 0.912 & 0.5 \\
Algas (A) & 0.526 & 0.0751 & 10.71 & $>0.001$ \\
Invertebrados (I) & 1.238 & 0.1768 & 12.05 & $>0.001$ \\
Sedimentos (S) & 0.163 & 0.0233 & 17.02 & $>0.001$ \\
\hline
\end{tabular}


MOPF

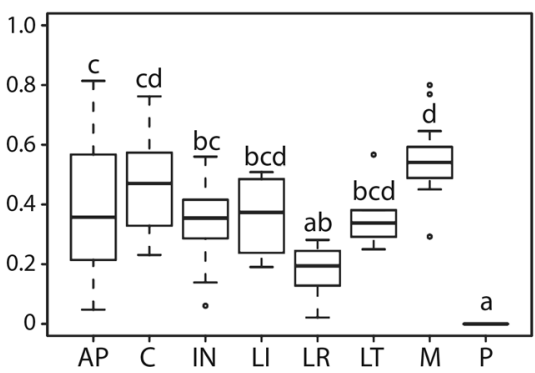

INVERTEBRADOS

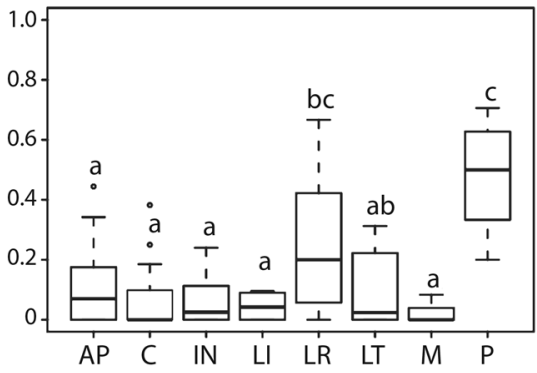

MOPG

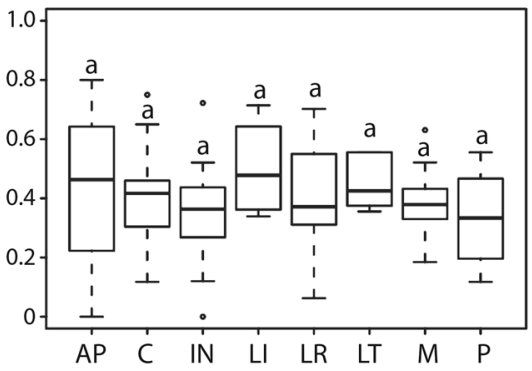

ALGAS

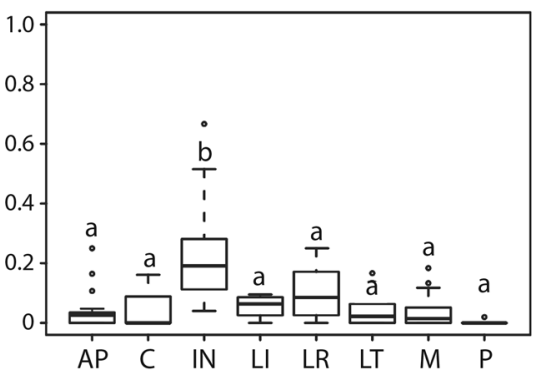

SEDIMENTO

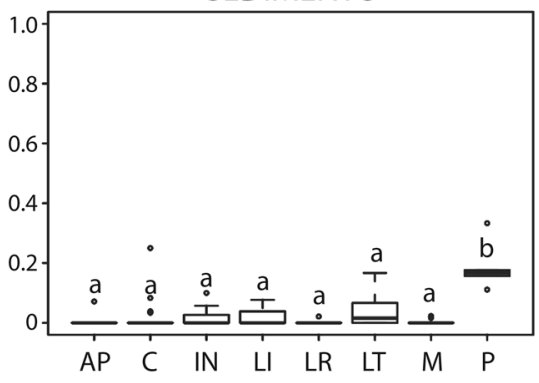

Fig. 3. Comparación de los ítems alimentarios considerados para cada sitio de estudio mediante análisis de Tukey. Las diferencias significativas $(\mathrm{P}<0.001)$ se representan con diferentes letras.

Fig. 3. Comparison of food items considered for each study site by the method of Tukey. Significant differences $(\mathrm{P}<0.001)$ are represented by different letters.

que se mantuvo con proporción constante independientemente del sitio analizado y no tuvo correlación con ningún eje ( $\mathrm{P}>0.05)$.

Los resultados del análisis de amplitud de nicho trófico revelaron diferencias en el uso de los recursos en los sitios estudiados (Fig. 5). En el sitio $M$ se obtuvo el valor de amplitud más bajo $\left(\mathrm{B}_{\mathrm{pop}}=0.27\right)$, denotando la dominancia de unos pocos recursos (MOPG y MOPF). Por otro lado, los mayores valores de amplitud se registraron en los sitios IN y LR
$\left(\mathrm{B}_{\mathrm{pop}}=0.349 \mathrm{y} \mathrm{B}_{\mathrm{pop}}=0.354\right.$, respectivamente $)$ en los que se encuentra una mayor diversificación en el uso de los recursos secundarios (algas e invertebrados).

De acuerdo al análisis presentado de piezas bucales y contenido estomacal, el GFT de Claudioperla tigrina es primariamente triturador, con la asignación secundaria que varía de acuerdo a la ecorregión considerada: recolector en Yungas y Pastizales de Neblina y depredador en Altos Andes. 


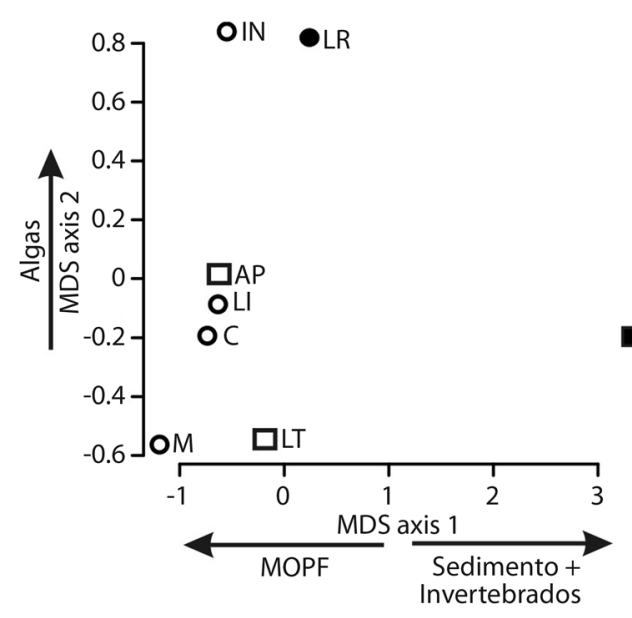

Fig. 4. Mapa bifactorial del ordenamiento de los sitios de acuerdo a los perfiles dietarios según análisis MDS. Altos Andes (घ), Pastizales de Neblina (०), Selva de Yungas ( $\square$ ) y Monte de Sierras y Bolsones $(\bullet)$.

Fig. 4. Bifactorial map of ordering of the sites according to the dietary profiles in MDS analysis. High Andes (๘), Humid Grasslands (०),Yungas Rainforest ( $\square$ ) and Argentine Northwest Monte and Thistle of the Prepuna $(\bullet)$.

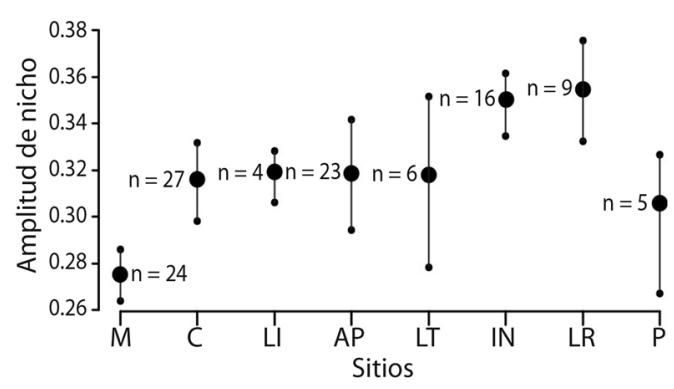

Fig. 5. Amplitud de nicho trófico calculado a partir de los perfiles dietarios correspondientes para cada sitio. Barras de extención correspondientes al $95 \%$ IC.

Fig. 5. Trophic niche breadth obtained from the dietary profiles for each site. Bars correspond to $95 \%$ CI.

\section{DISCUSIÓN}

Los antecedentes registrados sobre los hábitos alimentarios para la especie $C$. tigrina sólo la clasifican como herbívora (Romero, 2001). Nuestros resultados mostraron que las ninfas de $C$. tigrina ingirieron más de un ítem alimentario (MOPG, MOPF, invertebrados y algas), lo cual sugiere que se trata de una especie flexible en sus hábitos de alimentación. Los ejemplares de la clase de tamaño 1 (primeros estadios) presentan un consumo casi exclusivo de MOPF, comportándose como colectores. Merritt y Cummins (1996) sugieren que esta tendencia es común en los estadios tempranos y se debe a las limitaciones asociadas al tamaño reducido del aparato bucal. Consecuentemente, en las clases 2, 3 y 4 dominó la MOPG y el consumo de invertebrados también aumentó. La dieta cambia en relación al tamaño corporal ya que en los primeros estadios se consumen partículas más finas, mientras que en los estadios finales las partículas tienden a ser mayores (Lamberti \& Moore, 1984).

Cummins $(1973,1974)$ destacó la importancia de la morfología del aparato bucal como indicador del mecanismo de alimentación. Las mandíbulas y maxilas de C. tigrina, fuertes, esclerozadas y dentadas son probablemente las principales estructuras implicadas en la manipulación del alimento, su fragmentación e ingestión. Estas estructuras son similares a las descritas para Klapopteryx kuscheli (Plecoptera: Austroperlidae) asignada al grupo funcional de los trituradores (Albariño, 2001). Las setas presentes en las distintas partes de la boca parecen ser estructuras sensoriales y no estar involucradas en la ingesta de alimentos, como ocurre en otra especie de Gripopterygidae, Notoperla archiplatae, perteneciente al grupo funcional de los recolectores (Díaz Villanueva \& Albariño, 1999). Los fragmentos de hojas (MOPG) y algas contenidas en los estómagos mostraron signos de haber sido masticados. Esto junto con la fuerte esclerotización del aparato bucal, permite afirmar que la especie tiene hábitos trituradores. Dentro de la familia Gripopterygidae la especie Antarctoperla michaelseni fue asignada a dicho grupo funcional (Tierno de Figueroa, Vera \& López-Rodríguez, 2006); al igual que el género Paragrypopterix aunque secundariamente (Oliveira \& Nessimian, 2010). Esta asignación de C. tigrina al GFT de los trituradores puede no ser exclusiva. Por un lado la gran cantidad de MOPF presente podría dar indicios sobre la pertenencia secundaria al grupo de los colectores-recolectores 
como es el caso de la mayor parte de los géneros dentro de la familia Gripopterygidae, por ejemplo Paragripopteryx, Gripopteryx, Tupiperla y Guaranyperla (Oliveira \& Nessimian, 2010). Por otro lado, las proporciones de invertebrados encontradas en los tractos digestivos estarían marcando una tendencia a la depredación, lo cual parece constituir una novedad dentro de la familia en Sudamérica ya que en la bibliografía analizada no se encontraron asignaciones de gripopterígidos a este grupo funcional. Los ítems consumidos por $C$. tigrina mencionados anteriormente difieren de los encontrados por Froehlich y Oliveira (1997) en otros Gripopterygidae, Gripopteryx y Paragrypopteryx, cuyo principal componente fue perifiton y de las ninfas de Notoperla archiplatae, perteneciente a la misma familia, que ingieren principalmente diatomeas (Díaz Villanueva \& Albariño, 1999).

Las diferencias en la importancia de los ítems secundarios entre los distintos arroyos analizados puede deberse a la disponibilidad ambiental en las ecorregiones consideradas, que según Cummins (1984) puede representar una variabilidad adicional. En Yungas y Pastizales de Neblina, la vegetación de ribera está caracterizada por la presencia de árboles como Alnus acuminata. El procesamiento de las hojas como MOPG y posteriormente como MOPF, explicaría la disponibilidad de estos recursos como principal y secundario, respectivamente. Para estos sitios la asignación del GFT corresponde a triturador/recolector. Las regiones de Monte de sierras y bolsones e Infiernillo presentan en sus riberas gramíneas y arbustos, son arroyos abiertos que permiten el desarrollo de algas lo que explicaría su presencia en los contenidos estomacales como recurso secundario (Tomanova et al., 2006). Aunque Infiernillo se encuentra clasificado dentro de la ecorregión de Pastizal de Neblina, presenta un rango altitudinal semejante al sitio analizado para la ecorregión de Monte de sierras y bolsones (Inf $=2983 \mathrm{msnm}$; LR $=2851 \mathrm{msnm}$ ). El GFT asignado para dichos sitios es el de triturador/recolector. En la región de Altos Andes se registró la mayor altitud de muestreo (4467 msnm) y la presencia de gramíneas en la ribera. Como recurso principal consumido se encuentra la MOPG y secundariamente invertebrados, con registros de sedimento, lo que lleva a una designación de GFT de triturador/depredador.

La definición del GFT de C. tigrina y el establecimiento de su rol como procesador primario de materia orgánica representa un paso importante para futuros estudios de redes tróficas o aprovechar mejor el potencial analítico que pueden ofrecer las metodologías disponibles y basadas sobre grupos funcionales.

\section{AGRADECIMIENTOS}

A las personas e instituciones que donaron material o expidieron permisos de colecta en sus jurisdicciones: Daniel Emmerich, Carolina Nieto, José Rodríguez, Asambleas Ciudadanas Ambientalistas de La Rioja, Parque Nacional Los Alisos. Este trabajo se realizó en parte con el aporte de los siguientes subsidios a la investigación: PIP-CONICET 0330 y PICT1067 (Director: E. Domínguez).

\section{RESUMEN}

La determinación de los hábitos alimentarios permite la comprensión de muchos procesos ecológicos que se dan en los ecosistemas lóticos. El objetivo de este trabajo es: 1) conocer los hábitos alimentarios de ninfas de Claudioperla tigrina en un amplio gradiente altitudinal y latitudinal en Argentina; 2) asignar el grupo funcional trófico (GFT) de C. tigrina; 3 ) evaluar diferencias entre dietas de los sitios estudiados. Las ninfas estudiadas fueron recolectadas a lo largo de un amplio rango espacial en el noroeste de Argentina e incluyen diferentes estados del desarrollo (cuatro clases de tamaño). El material ingerido fue extraído de la parte anterior del tubo digestivo mediante disección ventral del tórax. Los perfiles dietarios fueron analizados a través de la estimación de parámetros de la distribución multinomial de Dirichlet. Se efectuaron ANOVA's para cada rubro alimentario en función de los sitios. Se utilizó la técnica Multidimensional Scaling (MDS) para identificar los sitios con perfiles alimentarios promedios similares. Se realizó un análisis de amplitud de nicho trófico para evaluar la diversificación de los recursos consumidos en cada sitio. El aparato bucal no muestra variaciones a lo largo del desarrollo ninfal, excepto una mayor esclerotización con la edad. Poseen palpos mandibulares y maxilares 
relativamente cortos y mandíbulas y maxilas fuertes, esclerozadas y dentadas. Las ninfas ingirieron más de un ítem alimentario, sugiriendo que se trata de una especie flexible en su alimentación. El ítem dominante en la dieta fue la MOPG, los signos de haber sido masticado indican hábitos trituradores. Se encontraron diferencias entre sitios para los ítems MOPF, invertebrados, algas y sedimento, pero no para MOPG. Se obtuvieron las correlaciones para cada eje del análisis MDS. Con el eje 1 se correlacionaron negativamente los sitios AP, LT, LI, C y M (Selva de Yungas y Pastizal de neblina) asociados a un mayor consumo de MOPF, en el lado positivo del eje se encontró el sitio P (Altos Andes) asociado a los ítems invertebrados y sedimento. Para el eje 2 se relacionaron positivamente IN (Pastizal neblina) y LR (Monte de Sierras y Bolsones) con la presencia de algas. Los resultados del análisis de amplitud de nicho revelaron diferencias en el uso de los recursos secundarios de la dieta en los sitios estudiados que puede deberse a la oferta ambiental en las ecorregiones consideradas. El GFT de C. tigrina es triturador/ recolector en Selva de Yungas y Pastizales de Neblina y triturador/ depredador en Altos Andes. La definición del GFT de $C$. tigrina y el establecimiento de su rol como procesador primario de materia orgánica representa un paso importante para futuros estudios de redes tróficas y otras metodologías basadas en los grupos funcionales.

Palabras clave: grupos funcionales tróficos, contenido estomacal, selva de Yungas, Pastizales de Neblina, Altos Andes, Monte de sierras y bolsones.

\section{REFERENCIAS}

Aitchison, J., \& Greenacre, M. (2002). Biplots of compositional data. Journal of the Royal Statistical Society: Series C (Applied Statistics), 51, 375-392.

Albariño, R. J. (2001). The food habits and mouthpart morphology of a South Andes population of Klapopteryx kuscheli (Plecoptera: Austroperlidae) aquatic insects. Aquatic Insects, 23, 171-181.

Albariño, R., \& Díaz Villanueva, V. (2006). Feeding Ecology of two Plecopterans in low order Andean- Patagonian streams. International Review of Hydrobiology, 91(2), 122-135.

Albariño, R. J., Díaz Villanueva, V., \& Canhoto, C. (2008). The effect of sunlight on leaf litter quality reduces growth of the shredder Klapopteryx kuscheli. Freshwater Biology, 53, 1881-1889.

Albariño, R. J. \& Valverde, A. (1998). Hábito alimenticio del estado larval de Parasericostoma cristatum (Trichoptera: Sericostomatidae). Revista de la Sociedad Entomológica Argentina, 57, 131-135.

Bello, C. L., \& Cabrera, M. I. (2001). Alimentación ninfal de Leptophlebiidae (Insecta: Ephemeroptera) en el
Caño Paso del Diablo, Venezuela. Revista de Biología Tropical, 49(3-4), 999-1003.

Castillo, G., Zúñiga, M. C., \& Bacca, T. (2013). El orden Plecoptera (Insecta) del departamento de Nariño, Colombia. Revista Colombiana de Entomología, 39(2), 229-236.

Cummins, K. W. (1973). Trophic relations of aquatic insects. Annual Review of Entomology, 18, 183-206.

Cummins, K. W. (1974). Structure and function of stream ecosystems. BioScience, 24, 631-641.

Cummins, K. W. (1984). Invertebrate food resource relationships. Bulletin of the North American Benthological Society, 1, 44-45.

De Cáceres, M., Sol, D., Lapiedra, O., \& Legendre, P. (2011). A framework for estimating niche metrics using the resemblance between qualitative resources. Oikos, 120, 1341-1350.

Díaz Villanueva, V., \& Albariño, R. J. (1999). Feeding habit of Notoperla archiplatae (Plecoptera) larvae in a North Patagonia Andean stream, Argentina. Hydrobiologia, 412, 43-52.

Fochetti, R., \& Tierno de Figueroa, J. M. (2008). Global diversity of stoneflies (Plecoptera; Insecta) in freshwater. Hydrobiologia, 595(1), 365-377.

Froehlich, C. G., \& Oliveira, L. G. (1997). Ephemeroptera and Plecoptera nymphs from riffles in loworder streams in southeastern Brazil. In P. Landolt, \& M. Sartori (Eds.), Ephemeroptera and Plecoptera: Biology-Ecology-Systematics (pp.180-185). Fribourg: MTL.

Gibon, F. M., \& Molina, C. I. (2013). Contribution to the Knowledge of the Andean Stonefly Genus Claudioperla Illies, with Description of New Apterous and Micropterous Species (Plecoptera: Gripopterygidae). Neotropical Entomology, 42(2), 170-177.

Illies, J. (1963). Revision der südamerikanischen Gripopterygidae (Plecoptera). Mittheilungen der Schweizer entomologischen Gesellschaft, 36, 145-248.

Klapálek, F. (1904). Plecopteren. In Ergebnisse, Hamburger Magalhaensische Sammelreise, 7(5), 1-13.

Klapálek, F. (1909) Vorläufige Bericht über exotische Plecopteren. Wiener Entomologische Zeitung, $28,215-232$.

Klapálek, F. (1914). Analytická tabulka fam. Perlidae a jeji dvou subfam. Perlinae a Acroneurinae (Plecoptera). Časopis České Spolecnosti Entomologické, 11, 53-69.

Lamberti, G. A., \& Moore, J. W. (1984). Aquatic insects as primary consumers. In V. H. Resh, \& D. M. Rosenberg (Eds.), The Ecology of Aquatic Insects (pp. 164195). New York: Praege. 
Merrit, R. W., \& Cummins, K. W. (1996). An Introduction to the Aquatic Insects of North America. Iowa: Kendall/Hunt.

Oliveira, A. L. H., \& Nessimian, J. L. (2010). Spatial distribution and functional feeding groups of aquatic insect communities in Serra da Bocaina streams, southeastern Brazil. Acta Limnologica Brasiliensia, 22(4), 424-441.

Olson, D. M., Dinerstein, E., Wikramanayake, E. D., Burgess, N. D., Powell, G. V. N., Underwood, E. C., D'amico, J. A., (...) \& Kassem, K. R. (2001). Terrestrial Ecoregions of the World: a new map of life on Earth. BioScience, 51, 933-938.

Pessacq, P. (2009). Estado de conocimiento de Plecoptera en Patagonia. Revista de la Sociedad Entomológica Argentina, 68(1-2), 169-175.

Reynaga, M. C. (2009). Hábitos alimentarios de larvas de Trichoptera (Instecta) de una cuenca subtropical. Ecología Austral, 19, 207-214.

Reynaga, M. C., \& Dos Santos, D. A. (2012). Rasgos biológicos de macroinvertebrados de ríos subtropicales: patrones de variación a lo largo de gradientes ambientales espacio-temporales. Ecología Austral, $22,112-120$.

Reynaga, M. C., \& Rueda Martín, P. (2008). Trophic analysis of two species of Atopsyche (Trichoptera: Hydrobiosidae). Limnologica, 40, 61-66.

Reynaga, M. C., \& Rueda Martín, P. (2014). Trophic analysis of three species of Marilia (Trichoptera: Odontoceridae) from the neotropics. Revista de Biologia Tropical, 62(2), 543-550.

Righi-Cavallaro, K. O., Froehlich, C. G., Cavallaro, M. R. (2014). Feeding Habits of Plecoptera Nymphs from the Atlantic Forest, Southeastern Brazil. Australian Journal of Basic \& Applied Sciences, 8(15), 226-232.
Romero, V. F. (2001). Plecoptera. In H. R. Fernández, \& E. Domínguez (Eds.), Guía para la determinación de los artrópodos bentónicos sudamericanos (pp.93-109). Tucumán: Facultad de Ciencias Naturales e Instituto Miguel Lillo.

Stark, B. P., Froehlich, C., \& Zúñiga, M. C. (2009). South American Stonflies (Plecoptera), In J. Adis, J. R. Arias, S. Golovatch, K. M. Wantzen, \& G. RuedaDelgado (Eds.), Aquatic Biodiversity of Latin American-ABLA (v. 5). Sofia-Moscow: Pensoft.

Stark, B. P., Kondratieff, B. C., \& Gill, B. (2012). Notes on Claudioperla tigrina (Klapálek) in Ecuador (Plecoptera: Gripopterygidae). Illiesia, 8, 141-146.

Tamarís-Turizo, C., Turizo-Correa, R., \& Zúñiga, M. C. (2007). Distribución espacio-temporal y hábitos alimentarios de ninfas de Anacroneuria (Insecta: Plecoptera: Perlidae) en el río Gaira (SNSM, Colombia). Caldasia, 29(2), 375-385.

Tierno de Figueroa, J. M., Vera, A., \& López-Rodríguez, M. J. (2006). Adult and Nymphal Feeding in the Stonefly species Antarctoperla michaelseni and Limnoperla jaffueli from Central Chile (Plecoptera: Gripopterygidae). Entomologia Generalis, 29(1), 39-45.

Tomanova, S., Goitia, E., \& Helesic, J. (2006). Trophic levels and functional feeding groups of macroinvertebrates in neotropical streams. Hydrobiologia, $556,251-264$.

Tvedebrink, T. (2015). Package 'dirmult': Estimation in Dirichlet-Multinomial distribution. Recuperado de http://cran.r-project.org/web/packages/dirmult/index. html

Wais, I. R. (1990). A checklist of the benthic macroinvertebrates of the Negro River Basin, Patagonia, Argentina, including an approach to their functional feeding groups. Acta Limnologica Brasiliensia, 3, 829-845. 